\title{
Gold nanorod-mediated photothermolysis induces apoptosis of macrophages via damage
}

\section{of mitochondria}

Aims: Induction of apoptosis or necrosis in activated macrophages by gold nanorod-mediated photothermolysis is demonstrated and the mechanisms underlying the processes are investigated. Materials \& methods: Gold nanorods were functionalized with cysteine-octaarginine peptides (R8-NRs). Uptake of R8-NRs by activated macrophages was monitored by two-photon luminescence imaging. The laser irradiation conditions were controlled to induce apoptosis or necrosis to R8-NR-internalized macrophages. Mitochondrial damage and reactive oxygen species overproduction during photothermolysis was investigated by confocal fluorescence microscopy and transmission-electron microscopy. Results: Activated macrophages efficiently uptake R8-NRs both in vitro and in live animals. Laser irradiation of internalized nanorods with controlled power density induces apoptosis of macrophages via intracellular perturbation and subsequent injury of mitochondria. Conclusions: Gold nanorod-mediated photothermolysis provides one promising way to eliminate activated macrophages in autoimmune and inflammatory diseases.

\section{KEYWORDS: apoptosis gold nanorods mitochondria damage necrosis photothermolysis primary activated macrophages two-photon luminescence imaging}

Macrophages constitute a major component of the mononuclear phagocyte system and play an important role in tissue homeostasis and inflammation. However, when macrophages become activated unnecessarily, they could contribute to many autoimmune and inflammatory diseases, such as atherosclerosis [1], rheumatoid arthritis [2] and sarcoidosis [3]. During the development of these diseases, activated macrophages may release cytokines, chemokines, digestive enzymes and prostaglandins to aggravate or accelerate damage to the normal tissues [4]. Therefore, detection and eradication of the macrophages in the injurious states provide an efficacious way to cure those diseases $[5,6]$. The distinctive function of macrophages to rapidly recognize and uptake particulate matter offers a preference to deliver nano-sized contrast agents (e.g., paramagnetic iron oxide nanocrystals [7]) and drug carriers (e.g., liposomes [8]) to macrophages. However, traditional drug carriers such as liposomes may induce considerable toxicity to healthy organs based on the biodistribution study [9]. A photomedicine method for selective eradication of activated macrophages with minimal toxicity to healthy organs would be attractive.

Optical hyperthermia has been used to eradicate diseased cells and tissues in a noninvasive manner. The photoactivated therapy could be localized and intensified by targeting exogenous agents with large absorption cross-sections to the area of interest and confining damage with minimal collateral effects. Gold nanospheres $[10-13]$, nanoshells $[14,15]$ and nanocages $[16,17]$ have been successfully used in ablation of tumor cells and tissues. Recently, gold nanorods have become an ideal probe for theragnosis (combined therapy and diagnosis) owing to their unique properties. They can be easily synthesized $[18,19]$ and modified with various surface modifications including antibody [20-23], surfactant [24], folic acid [25,26], phospholipid [27], DNA [28], peptide [29] and polymer [24,30-32]. Unlike gold nanospheres, the longitudinal plasmon resonance mode of nanorods could be tuned to the near-infrared region, a spectral window permitting relatively deep tissue penetration of photons. Besides a strong plasmon scattering [33,34], it has recently been shown that gold nanorods emit a bright two-photon luminescence (TPL) [35,36], allowing 3D imaging of nanorod-labeled cells $[23,24]$. Meanwhile, more than $96 \%$ of the absorbed photons in nanorods are converted into heat by the ultra-fast nonradiative electron relaxation dynamics [37], at a higher efficiency than gold nanospheres and nanoshells [38-40]. These properties make gold nanorods an attractive agent for gene delivery $[28,41]$, cell imaging [22-24,42] and photothermolysis of tumor cells $[25,26,39,43]$, macrophage cell lines [44] and pathogens [45,46].

In nearly all the photothermal injury studies, cell death was determined by checking the cell membrane permeability with fluorescent

Ling Tong' ${ }^{\text {\& }}$
Ji-Xin Cheng',2†
'Author for correspondence:
${ }^{1}$ Department of Chemistry,
Purdue University,
West Lafayette, IN, USA
${ }^{2}$ Weldon School of
Biomedical Engineering,
Purdue University,
West Lafayette,
IN 47907, USA
Tel.: +1 $7654944335 ;$
Fax: +1 765496 1912;
E-mail: jcheng@purdue.edu

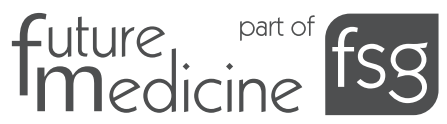


indicators, such as trypan blue [39,43], calcein AM [26] and ethidium bromide [25,26]. No control of cell death in either the necrosis or apoptosis pathway has been considered. However, apoptosis is preferred for in vivo applications because necrosis might induce serious secondary inflammation. In addition, despite the report of membrane damage by irradiation of nanoparticles $[10,12]$ and gold nanorods [26], the mechanisms underlying intracellular injury remain under-investigated.

In this article, we demonstrate the use of nanorod-mediated optical hyperthermia for elimination of primary activated macrophages. We show that gold nanorods functionalized with arginine-rich peptides could be selectively and efficiently internalized by activated macrophages in vitro and in vivo. Moreover, we demonstrate that controlled laser irradiation of internalized nanorods could lead to apoptosis by internal damage of mitochondria or acute necrosis by compromising the plasma membrane integrity.

\section{Materials \& methods \\ Preparation \& characterization of R8-NRs}

Gold nanorods were synthesized in micellar surfactant (cetyl trimethyltrimethylammonium bromide $[\mathrm{CTAB}]$ ) solution using seeded growth method with the presence of $\mathrm{AgNO}_{3}[18,47]$. The growth was initiated 10-15 min after seed injection, carefully monitored by an UV-visible spectrophotometer (DU-530, Beckman, CA, USA) for the next 5-30 min and quenched at the desired wavelength with 15 min treatment of $\mathrm{Na}_{2} \mathrm{~S}$ [47]. The nanorods were then centrifuged $(10,500 \mathrm{rpm}$ for $10 \mathrm{~min}$ ) to remove residual CTAB and sulfide and redispersed in milli-Q water, resulting in an optical density (O.D.) of 1.5-2.0. Particle size analysis by transmission-electron microscopy (TEM) indicated a mean length and aspect ratio of $45.5 \mathrm{~nm}$ and 3.8, respectively.

R8-NRs were prepared by conjugation of cysteine-octaarginine peptides (GenScript, NJ, USA) through an Au-S bond. Briefly, gold nanorod solution was centrifuged at $10,000 \mathrm{rpm}$ for $6 \mathrm{~min}$ to remove unassociated CTAB and re-suspended in milli-Q water. A total of $2.5-\mathrm{ml}$ nanorod solution (O.D. $~ 1.8$ ) was treated with a dropwise addition of $200 \mu \mathrm{l}$ aqueous solution containing $0.5 \mathrm{mg}$ peptides. The mixture was kept at room temperature overnight and dialyzed (MWCO 6000-8000, Fisher Scientific, PA, USA) for $12 \mathrm{~h}$ to remove CTAB and unconjugated peptides. This procedure yielded a stable dispersion of R8-NRs with a plasmon resonance peak at $788 \mathrm{~nm}$ and a final O.D. close to 1.3. The extinction spectra of the nanorods were not affected by surface modification, as shown in Figure 1B. The nanorod concentration was estimated using the measured extinction coefficient $(\varepsilon=4.6 \pm$ $0.6 \times 10^{9} \mathrm{M}^{-1} \mathrm{~cm}^{-1}$ ) [48], to be 0.28 and $0.22 \mathrm{nM}$ for O. D. of 1.3 and 1.0, respectively. To determine the binding efficiency of peptide on the nanorod surface, we performed an amino acid analysis to measure the amount of peptides conjugated to gold nanorods. The analysis gave $73 \mathrm{nmol}$ peptides for $3.94 \mathrm{pmol}$ nanorods, corresponding to $1.85 \times 10^{4}$ peptides per nanorod. PEGylated nanorods (mPEG-NRs) were prepared by a procedure similar to R8-NRs, with the addition of $3 \mu \mathrm{mol}$ of $\mathrm{mPEG}_{5 \mathrm{~K}}-\mathrm{SH}$ (Rapp Polymere GmbH, Tübingen, Germany; aqueous solution, $\mathrm{pH}=9$ ) to $4 \mathrm{ml}$ of nanorod solution.

\section{Recruitment of peritoneal macrophages \& cellular uptake of NRs}

Dehydrated Brewer thioglycollate medium powder (Sigma-Aldrich, MO, USA) was dissolved in water $(29.8 \mathrm{~g} / \mathrm{l})$ and autoclaved for $20 \mathrm{~min}$ at $15 \mathrm{lbs}$ pressure $\left(121^{\circ} \mathrm{C}\right)$. The autoclaved medium was kept in the dark under sterile conditions at room temperature for 3 months before use. Primary peritoneal macrophages were elicited by intraperitoneal injection of $1.5 \mathrm{ml}$ of the sterile thioglycollate medium into BALB/c mice. On the fourth day, after euthanizing the mouse by $\mathrm{CO}_{2}$, macrophages were collected by peritoneal lavage using $8 \mathrm{ml}$ phosphate-buffered saline (PBS), plated into the glass-bottomed Petri dishes (MatTek, MA, USA) in RPMI 1640 medium (Invitrogen, CA, USA) with 10\% fetal bovine serum (FBS; Sigma-Aldrich) and $1 \%$ penicillin-streptomycin (Invitrogen) for $2 \mathrm{~h}$ and washed with PBS three times to remove nonadherent cells. Then the macrophages were treated with an aliquot of R8-NRs $(100 \mu \mathrm{l}$, $0.22 \mathrm{nM}$ ) or CTAB-coated nanorods (CTABNRs, $100 \mu \mathrm{l}, 0.22 \mathrm{nM})$ or mPEG-NRs $(100 \mu \mathrm{l}$, $0.22 \mathrm{nM}$ ), and incubated with periodic monitoring. For in vivo uptake experiments, $300 \mu \mathrm{l}$ of R8-NR solution $(0.22 \mathrm{nM})$ was injected into the peritoneal cavity of a mouse prestimulated with thioglycollate medium. The mouse was then euthanized and the peritoneal macrophages were collected at $0.5 \mathrm{~h}$ after injection, centrifuged at $1000 \mathrm{rpm}$ for $10 \mathrm{~min}$ to remove free R8-NRs and plated on the Petri dish. Tri-colorlabeled F4/80 antibody (1:200, Invitrogen) was used to mark macrophages. 


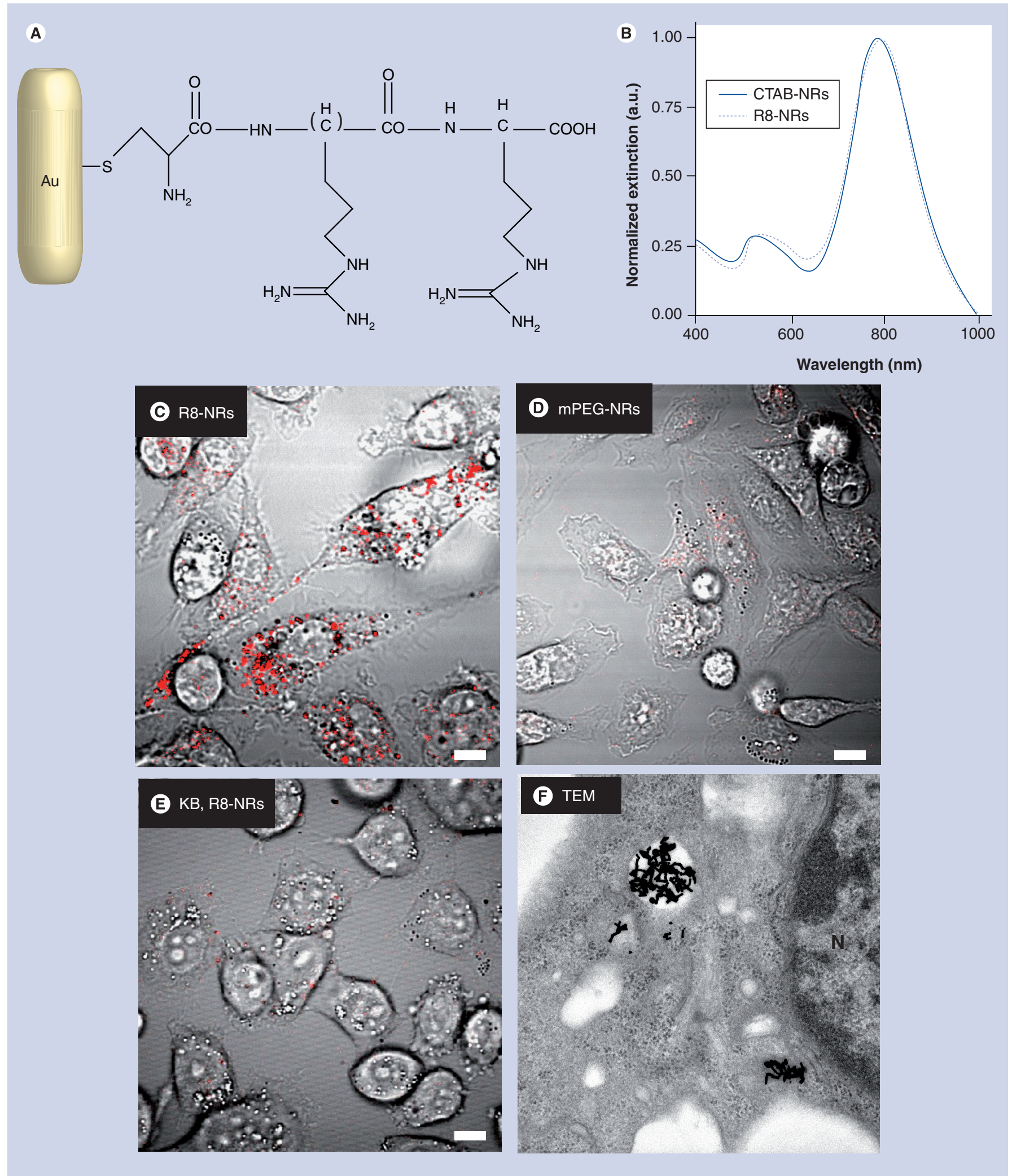

Figure 1. In vitro uptake of R8-NRs by macrophages. (A) Structure of R8-NR. (B) Normalized extinction spectra of gold nanorods before and after conjugation with peptides. (C) R8-NRs (red) were efficiently internalized by primary activated macrophages. (D) Only a few mPEG-NRs entered activated macrophages. (E) Only a few R8-NRs entered KB cells. Scale bar: $10 \mu \mathrm{m}$. (F) TEM image (285000x) of R8-NRs inside a macrophage. For (C-F), macrophages or KB cells were incubated with 100- $\mu \mathrm{l}$ R8-NR solution for $3 \mathrm{~h}$ at $37^{\circ} \mathrm{C}$. a.u.: Arbitrary unit; CTAB-NR: Cetyl trimethyltrimethylammonium bromide gold nanorod; mPEG-NR: PEGylated gold nanorod; N: Nucleus; R8-NR: Cysteine-octaarginine-conjugated gold nanorod; TEM: Transmission-electron microscopy. 


\section{Cell culture}

$\mathrm{KB}$ cells (a carcinoma cell line) and Chinese hamster ovary $(\mathrm{CHO})$ cells were cultured at $37^{\circ} \mathrm{C}$ in a humidified atmosphere containing $5 \% \mathrm{CO}_{2}$ and grown continuously in folatedeficient RPMI 1640 medium containing $10 \%$ FBS and $1 \%$ penicillin-streptomycin. In a typical experiment, a $1-\mathrm{ml}$ suspension of $\mathrm{KB}$ cells or $\mathrm{CHO}$ cells $\left(10^{5} / \mathrm{ml}\right)$ was plated onto a glass-bottomed Petri dish, grown for 2 days, then treated with an aliquot of R8-NRs $(100 \mu \mathrm{l}, 0.22 \mathrm{nM})$ and maintained at $37^{\circ} \mathrm{C}$ with periodic monitoring.

\section{TPL \& TEM imaging}

A femtosecond (fs) Ti:sapphire laser (MaiTai HP, Spectra-Physics, CA, USA) with a pulse width of $130 \mathrm{fs}$ and a repetition rate of $80 \mathrm{MHz}$ was used for TPL imaging of nanorods. The laser beam was directed into a scanning confocal microscope (FV1000/IX81, Olympus America Inc., CA, USA) equipped with a $60 \times$ water-immersion objective $(\mathrm{NA}=1.2)$. The excitation power used was $0.7 \mathrm{~mW}$ at the sample. TEM images were acquired at Purdue University Life Science Microscopy facility (IN, USA) following standard procedures.

\section{Controlled laser irradiation of macrophages loaded with R8-NRs}

Macrophages incubated with R8-NRs were rinsed with fresh RPMI 1640 medium and irradiated with the Ti:sapphire laser beam with an expanded diameter of $5 \mathrm{~mm}$ at $788 \mathrm{~nm}$ for $15 \mathrm{~min}$. The laser power was increased from
$0.43 \mathrm{~W}\left(2.18 \mathrm{~W} / \mathrm{cm}^{2}\right)$ to $0.86 \mathrm{~W}\left(4.36 \mathrm{~W} / \mathrm{cm}^{2}\right)$. The macrophages were kept at $37^{\circ} \mathrm{C}$ during the irradiation to mimic the physiological environment. Control experiments were performed at the same condition with macrophages without R8-NRs.

\section{Analysis of cell death}

Cell death was determined using a standard apoptosis kit (Invitrogen) including Alexa Fluor 488 annexin $\mathrm{V}$ to indicate early apoptosis and propidium iodide (PI) to label necrosis. A total of $5 \mu$ l of Alexa Fluor 488 annexin V and $1 \mu \mathrm{l}$ of PI $(100 \mu \mathrm{g} / \mathrm{ml})$ were added to macrophages in $100 \mu \mathrm{l}$ annexin-binding buffer and incubated at room temperature for $15 \mathrm{~min}$. As a positive control, paclitaxel (PTX)-induced apoptosis was performed by treatment of macrophages with $1 \mu \mathrm{MPTX}$ for $6 \mathrm{~h}$ at $37^{\circ} \mathrm{C}$. For quantitative analysis of cell death at different laser powers, the macrophages were plated in a 96-well plate with $100 \mu \mathrm{l}$ RPMI medium in each well and treated with the R8-NR solution $(0.22 \mathrm{nM}, 10 \mu \mathrm{l}$ for each well). The percentage of necrotic or apoptotic cells was determined by dividing the number of macrophages labeled with both PI and Alexa Fluor 488 annexin V or Alexa Fluor 488 annexin V alone, with the total number of macrophages counted in the well (>1300). A MTT assay was also performed to quantify the cell death. After laser irradiation, $10 \mu \mathrm{l}$ MTT solution $(5 \mathrm{mg} / \mathrm{ml}$ in PBS) was added to each well and incubated at $37^{\circ} \mathrm{C}$ for $3 \mathrm{~h}$. After removing the medium, $200 \mu \mathrm{l}$ dimethyl sulfoxide was added to each well and
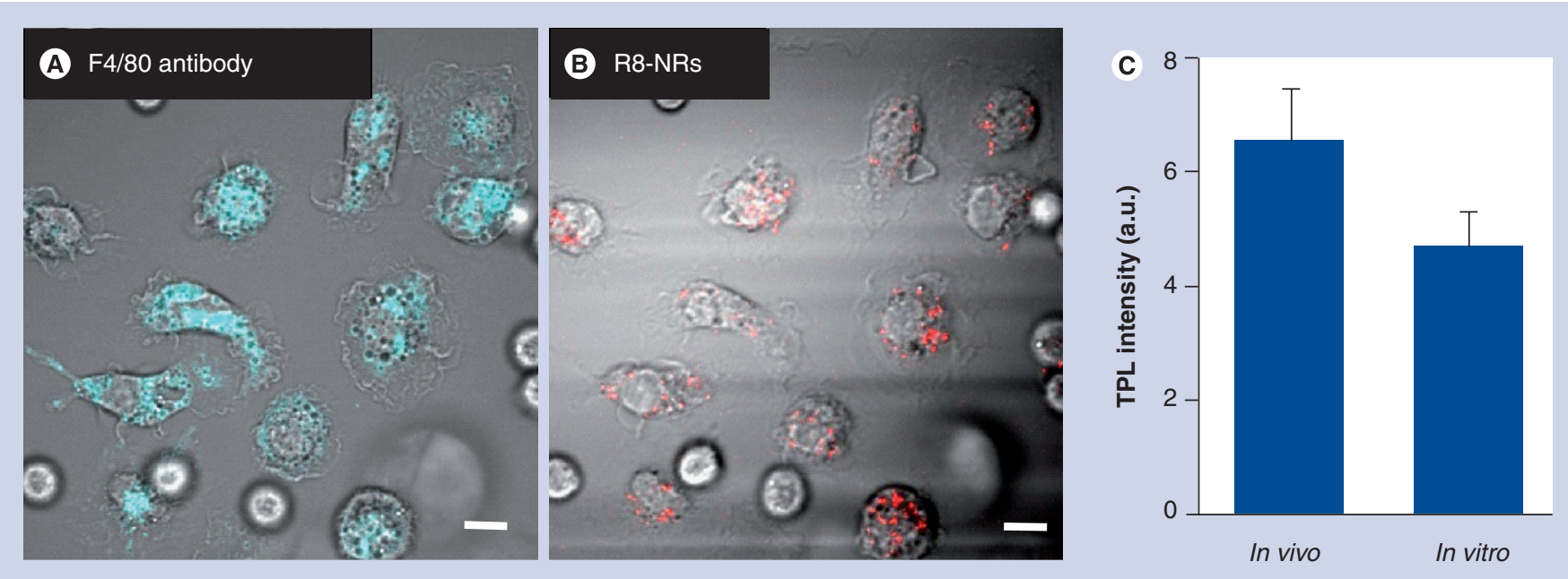

Figure 2. In vivo uptake of R8-NRs by macrophages at $\mathbf{0 . 5} \mathbf{~ h}$ after intraperitoneal injection. (A) Confocal fluorescence image of Tri-color-conjugated F4/80 antibody (blue) labeled macrophages. (B) TPL image of R8-NRs (red) in macrophages. Only F4/80+ macrophages had R8-NR labeling. (C) Statistical analysis of intracellular TPL intensity from R8-NRs in 50 macrophages randomly chosen from in vitro and in vivo experiments within $0.5 \mathrm{~h}$ incubation. Scale bar: $10 \mu \mathrm{m}$.

a.u.: Arbitrary unit; R8-NR: Cysteine-octaarginine-conjugated gold nanorod; TPL: Two-photon luminescence. 


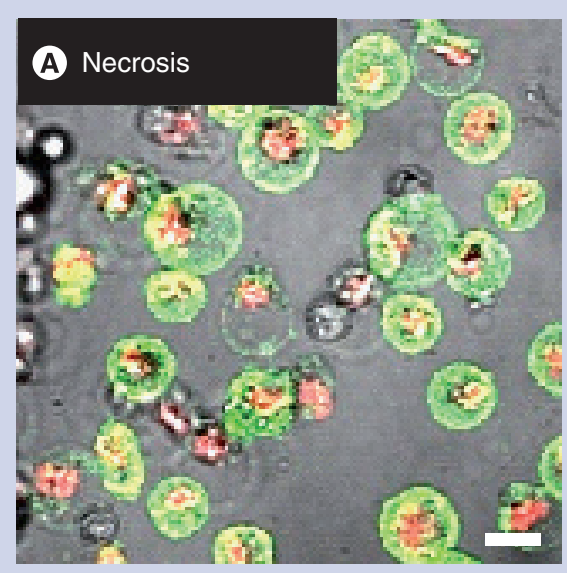

D

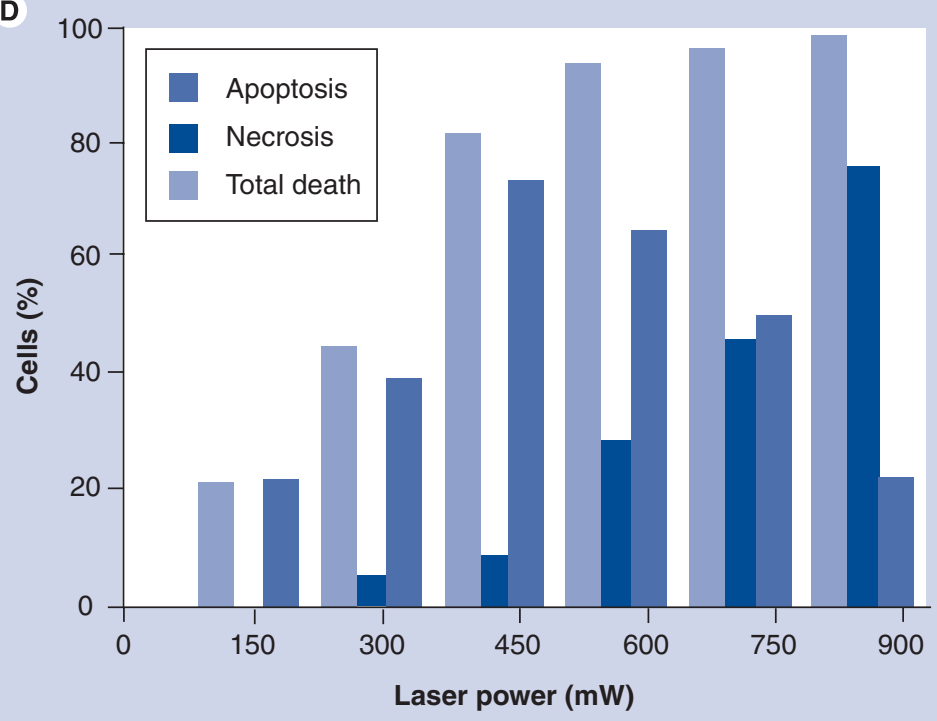

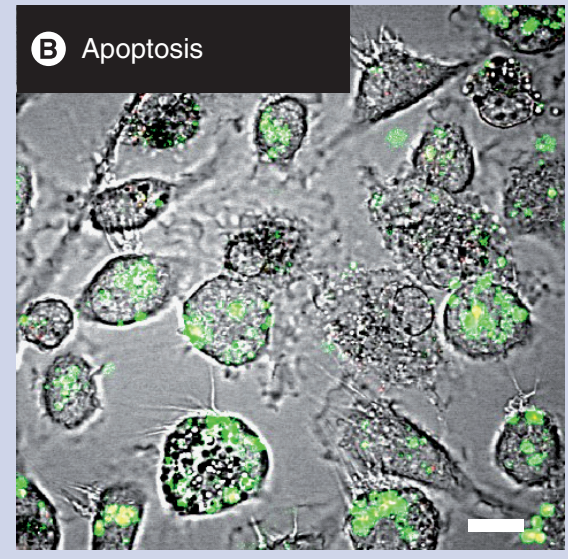

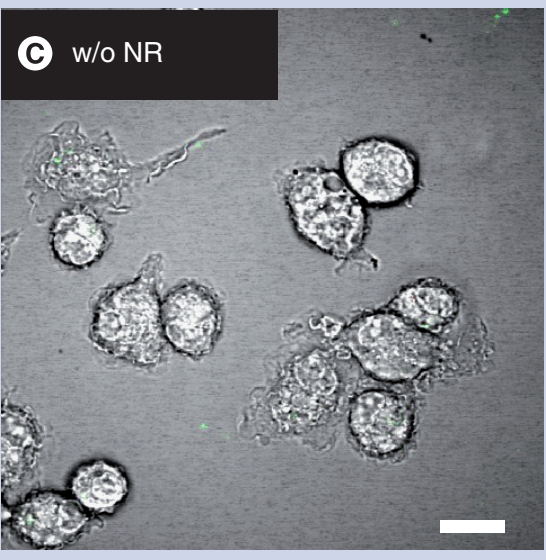

E

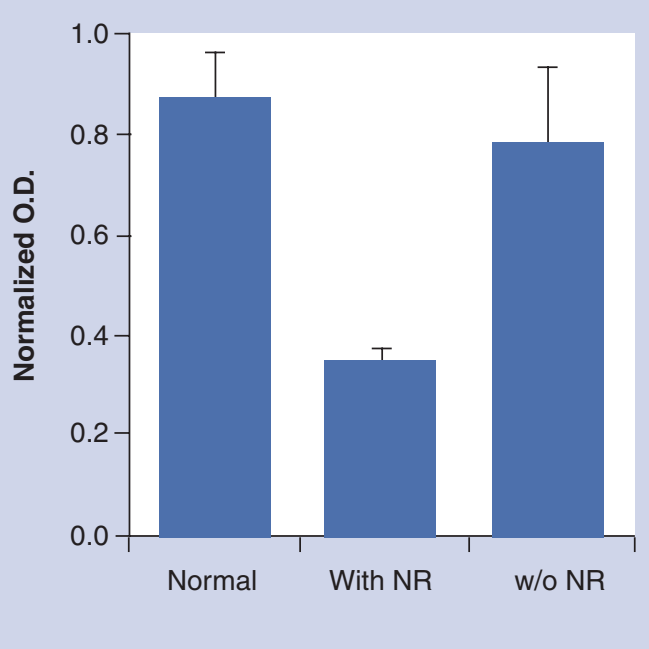

Figure 3. Controlled induction of apoptosis and necrosis to macrophages. (A) Necrosis of R8-NR-internalized macrophages induced by irradiation at $4.36 \mathrm{~W} / \mathrm{cm}^{2}$, indicated by Alexa Fluor 488 annexin V (green) and propidium iodide (red). (B) Apoptosis of R8-NR internalized macrophages induced at $2.18 \mathrm{~W} / \mathrm{cm}^{2}$, indicated by Alexa Fluor 488 annexin $\mathrm{V}$ (green). (C) Negative control. Macrophages without R8-NRs did not enter apoptosis under the same condition with (B). Scale bar: $10 \mu \mathrm{m}$. (D) Percentage of apoptosis and necrosis at different laser powers $(\mathrm{mW})$. (E) MTT assay result. Normal: macrophages without NR and laser irradiation; W/NR: NR internalized macrophages irradiated at $2.18 \mathrm{~W} / \mathrm{cm}^{2}$; w/O NR: Macrophage irradiated at $2.18 \mathrm{~W} / \mathrm{cm}^{2}$.

NR: Nanorod; O.D.: Optical density; R8-NR: Cysteine-octaarginine-conjugated gold nanorod.

the O.D. was read at $570 \mathrm{~nm}$ using a spectrophotometer (SpectraMAX 190, Molecular Devices Corp., CA, USA). Data from eight wells were analyzed for each group.

\section{Mechanism study}

To examine the plasma membrane integrity and $\mathrm{Ca}^{2+}$ influx in apoptosis induced at lower laser power density and necrosis induced at higher laser power density, Oregon Green BAPTA 2 (Invitrogen) was added to macrophages with a final concentration of $4 \mu \mathrm{M}$ and washed with medium before laser irradiation. For the mitochondria damage study, Mito Tracker Red CMXRos (membrane potential dependent,
Invitrogen) or Mito Tracker Green FM (membrane potential independent, Invitrogen) was added to macrophages with a final concentration of $0.12 \mu \mathrm{M}$. To detect the level of reactive oxygen species, carboxy- $\mathrm{H}_{2}$ DCFDA (Invitrogen) was added to macrophages after irradiation with a final concentration of $25 \mu \mathrm{M}$ and incubated at $37^{\circ} \mathrm{C}$ for $30 \mathrm{~min}$. Fluorescence imaging was carried out on a confocal microscope; 488-nm $\mathrm{Ar}^{+}$laser and 543-nm He/Ne laser were used for excitation with 10.0 and $9.5 \mu \mathrm{W}$ at the sample, respectively. Fluorescence intensities of Mito Tracker Red or Oregon Green BAPTA 2 within more than 100 randomly selected macrophages were measured for statistical analysis. 


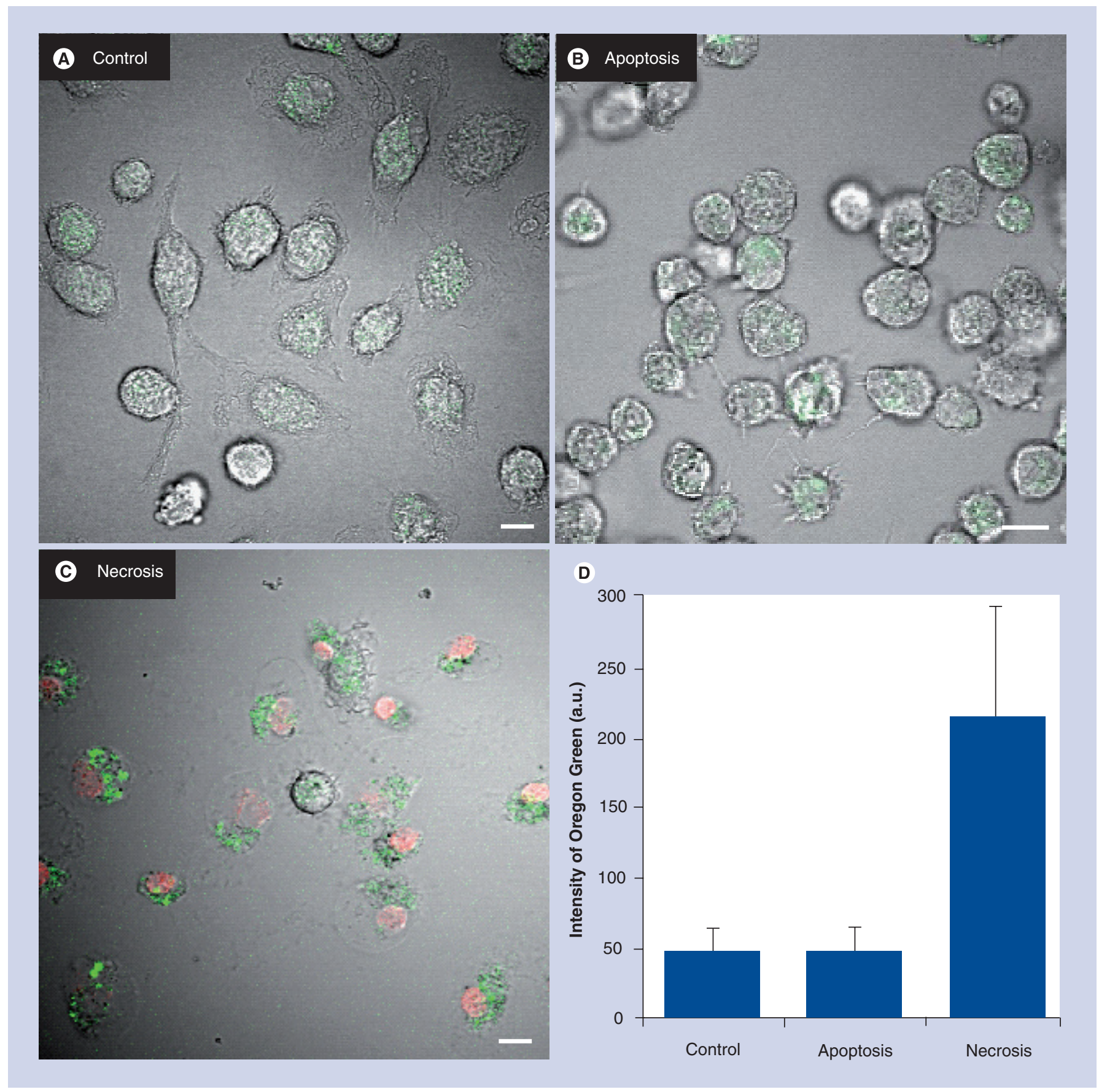

Figure 4. Examination of $\mathrm{Ca}^{2+}$ influx in apoptotic and necrotic macrophages under low and high power density. Macrophages were incubated with $\mathrm{Ca}^{2+}$ indicator Oregon Green BAPTA $2(4 \mu \mathrm{M})$ for 25 min and washed before irradiation. (A) Control: macrophages after irradiation at $2.18 \mathrm{~W} / \mathrm{cm}^{2}$. (B) Apoptosis: nanorod (NR)-internalized macrophages after irradiation at $2.18 \mathrm{~W} / \mathrm{cm}^{2}$. (C) Necrosis: NR-internalized macrophages after irradiation at $4.36 \mathrm{~W} / \mathrm{cm}^{2}$. Necrosis was indicated by propidium iodide (red). (D) Statistical analysis of fluorescent intensity of intracellular Oregon Green at different conditions. Scale bar: $10 \mu \mathrm{m}$. a.u.: Arbitrary unit.

\section{Results \& discussion}

Gold nanorods with a length of $45.5 \mathrm{~nm}$ and aspect ratio of 3.8 were synthesized in micellar surfactant $(\mathrm{CTAB})$ solution using the seeded growth method in the presence of $\mathrm{AgNO}_{3}$ $[18,47]$. To replace the cytotoxic surfactant $\mathrm{CTAB}$ on the nanorod surface and promote the
\end{abstract}

cellular internalization, gold nanorods were conjugated with cell-penetrating peptides, cysteineoctaarginine, to produce R8-NRs (Figure 1A). The surface modification with a coating of $1.85 \times 10^{4}$ peptides per nanorod yielded a stable dispersion of gold nanorods without any change of the extinction spectra profile and the 
plasmon resonance peak at $788 \mathrm{~nm}$ (Figure 1B). Monitored by TPL imaging, the R8-NRs were found inside primary macrophages after $0.5 \mathrm{~h}$ incubation and the cellular uptake increased with time (Supplementary Figure 1A-C) (see online www.futuremedicine.com/toc/nnm/4/3). The efficiency was much higher than CTAB-NRs (Supplementary Figure 1D). After $3 \mathrm{~h}$ incubation, a high density of R8-NRs was found in the cytoplasm (Figure 1C). By contrast, few mPEG-NRs were observed after $3 \mathrm{~h}$ incubation (Figure 1D). Additionally, the uptake of R8-NRs in macrophages was much faster than other cell types. Only a few R8-NRs were found binding to KB cells (Figure 1E) or CHO cells (not shown) after $3 \mathrm{~h}$ incubation. The inefficient internalization in $\mathrm{KB}$ or $\mathrm{CHO}$ cells was likely due to the large size of the functionalized gold nanorods compared with clathrin-coated pits, which slowed down the uptake. On the other hand, the cationic arginine peptides could conceivably bind to the anionic components on the macrophage surface (e.g., cysteine-rich domain of mannose receptors) and trigger phagocytic uptake of nanorods. The phagocytic internalization of gold nanorods in macrophages was supported by TEM (Figure 1F), where an accumulation of nanorods was found in the cytoplasmic vesicles. The cytotoxicity of R8-NRs in macrophages was examined by using calcein AM to indicate the viability and ethidium bromide staining of nuclei to indicate increased membrane permeability. After $32 \mathrm{~h}$ incubation with R8-NRs, the macrophages were strongly labeled with calcein AM, whereas the ethidium bromide signal was undetectable, showing little cytotoxicity of nanorods (Supplementary Figure 2).
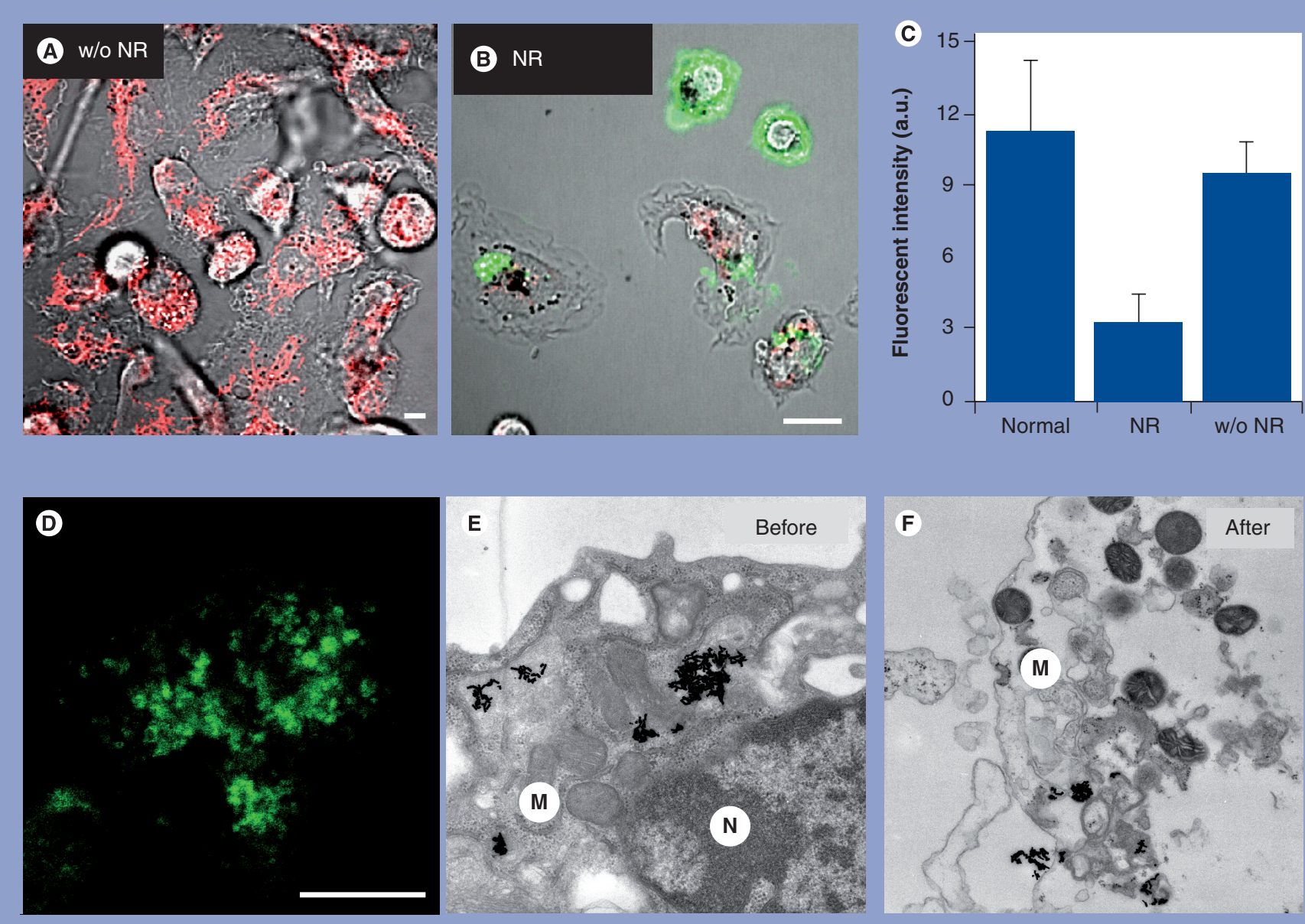

Figure 5. Mitochondria damage during apoptosis. The laser irradiation was at $2.18 \mathrm{~W} / \mathrm{cm}^{2}$ for 15 min in all experiments.

(A-C) Decrease of mitochondrial membrane potential. Mitochondria were labeled by Mito Tracker Red CMXRos (0.12 $\mu$ M). Apoptotic macrophages were indicated by Alexa Fluor 488 annexin V (green). (A) Macrophages without R8-NRs did not undergo apoptosis and were strongly labeled with Mito Tracker Red. (B) R8-NR-internalized macrophages were apoptotic (green) and the labeling of Mito Tracker Red significantly reduced. (C) Fluorescent intensity of intracellular Mito Tracker Red dropped 70\% in R8-NR-internalized macrophages and $16.8 \%$ in macrophages without NRs. (D) Mitochondria swelling (labeled with Mito Tracker Green FM) after laser irradiation. Scale bar: $10 \mu \mathrm{m}$. (E \& F) Transmission-electron microscopy images before and after laser irradiation (×285000) also indicated mitochondria swelling. a.u.: Arbitrary unit; M: Mitochondria; N: Nucleus; NR: Nanorod; R8-NR: Cysteine-octaarginine-conjugated gold nanorod. 

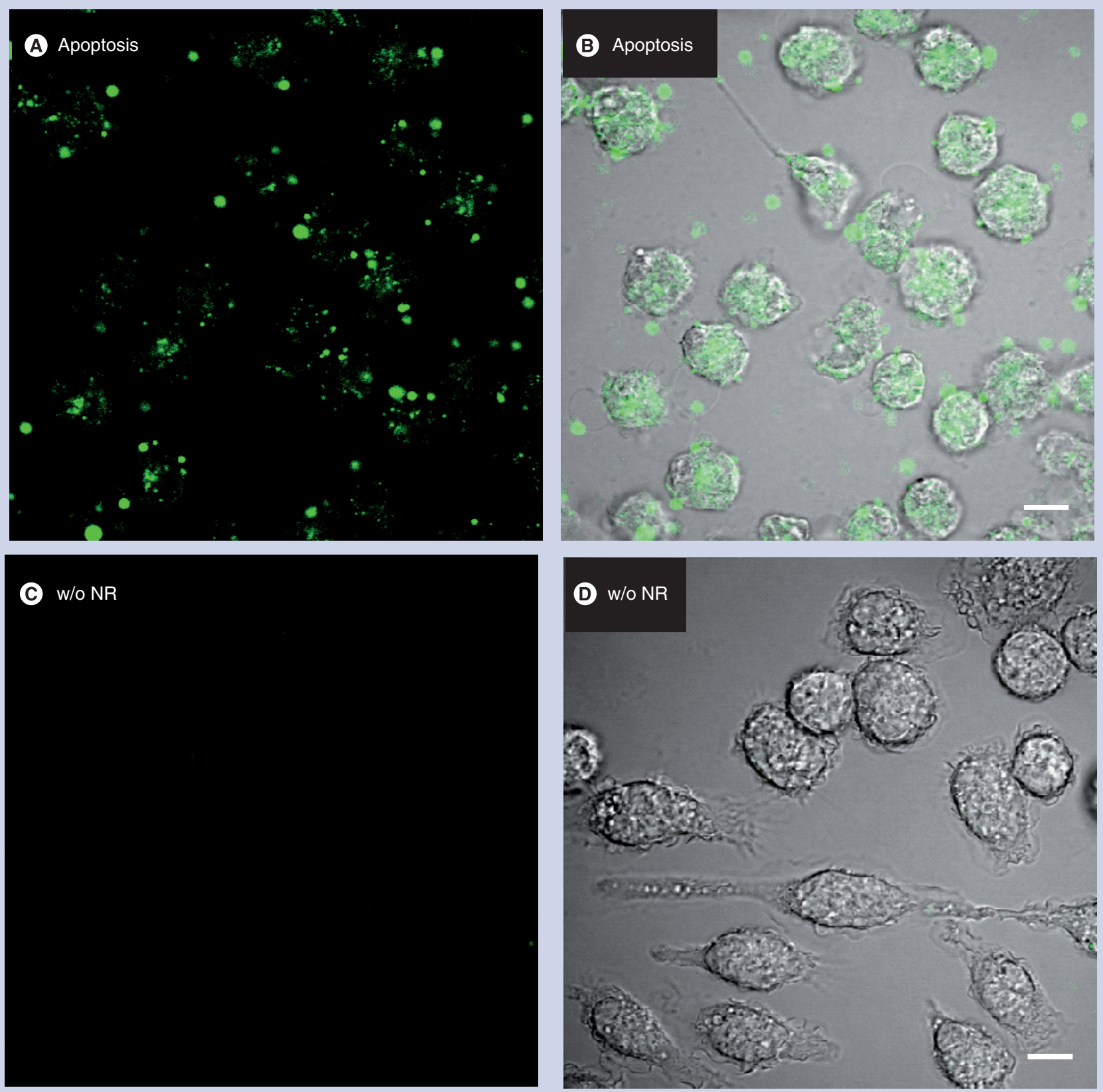

Figure 6. Reactive oxygen species overproduction during nanorod-mediated photothermalysis. After laser irradiation at $2.18 \mathrm{~W} / \mathrm{cm}^{2}$ of macrophages with (A \& B) or without (C \& D) NRs, reactive oxygen species (ROS) indicator (carboxy- $\mathrm{H}_{2}-\mathrm{DCFDA}$; green) was added and incubated for 30 min. (A \& B) ROS was detected (green) in apoptotic macrophages with NRs after irradiation.

(C \& D) No ROS was detected in macrophages without NRs at the same condition. Scale bar: $10 \mu \mathrm{m}$.

NR: Nanorod.

To test whether R8-NRs could target macrophages in vivo, $300 \mu \mathrm{l}$ of R8-NR solution $(0.22 \mathrm{nM})$ was directly injected into the peritoneal cavity of a mouse prestimulated with thioglycollate. After $0.5 \mathrm{~h}$, the mouse was euthanized and peritoneal macrophages were collected by lavage. Tri-color-conjugated F4/80 antibody was used to mark the activated macrophages. After washing the macrophages with PBS, F4/80 labeling and R8-NR internalization were examined by sequential confocal fluorescence and TPL imaging. As shown in Figure 2A \& B, only F4/80-positive cells showed R8-NR labeling. The small round cells (presumable neutrophils) did not uptake any R8-NRs. Compared with the in vitro results, higher in vivo uptake efficiency was found during the short $0.5 \mathrm{~h}$ incubation period (Figure 2C). The 
selective internalization allows subsequent photothermolysis of activated macrophages by laser irradiation of internalized nanorods.

We first optimized the laser irradiation conditions to induce apoptosis or necrosis to R8-NRinternalized macrophages by changing the power of an $\mathrm{fs}$ laser beam with a beam diameter of $5 \mathrm{~mm}$. The laser wavelength was tuned to $788 \mathrm{~nm}$, corresponding to the longitudinal plasmon peak of the nanorods used. Alexa Fluor 488 annexin V (green), a phosphatidylserine indicator, was used to label the plasma membrane of apoptotic cells and PI (red) was used to stain the nuclei of necrotic cells. We counted the macrophages that underwent apoptosis and necrosis at different laser powers (Figure 3D). When the laser power was as high as $0.86 \mathrm{~W}$ (power density $4.36 \mathrm{~W} /$ $\mathrm{cm}^{2}$ ), $98 \%$ of nanorod-internalized macrophages were damaged after $15 \mathrm{~min}$ irradiation, and $76 \%$ underwent necrosis, with both membranes (green) and nuclei (red) stained (Figure 3A). By reducing the laser power, the percentage of necrotic cells decreased. At the power density of $2.18 \mathrm{~W} / \mathrm{cm}^{2}$ $(0.43 \mathrm{~W}), 73 \%$ of the macrophages were apoptotic and were only labeled by Alexa Fluor 488 annexin $\mathrm{V}$ on the membrane (green, Figure $3 \mathbf{B}$ ). After further incubation at $37^{\circ} \mathrm{C}$ for $30 \mathrm{~h}$, the macrophages underwent late apoptosis, stained with PI (Supplementary Figure 3A \& B). The laser power density was lower than the one used in the earlier studies with gold nanorods $\left(10 \mathrm{~W} / \mathrm{cm}^{2}\right)$ [39] and nanoshells $\left(35 \mathrm{~W} / \mathrm{cm}^{2}\right)$ [14], possibly because of the efficient uptake of R8-NRs by macrophages and the use of an fs laser. The fs pulses were shown to be more effective than a continuous-wave laser in the optical-thermal transition for gold nanoparticles [26]. As a negative control, macrophages without nanorods were irradiated under the same condition. Little staining of Alexa Fluor 488 annexin $\mathrm{V}$ was observed (FIGURE 3C) and the macrophages remained viable after further incubation for $30 \mathrm{~h}$. As a positive control, macrophages were treated with $1 \mu \mathrm{M}$ PTX, a drug known to induce apoptosis [49], for $6 \mathrm{~h}$. It was found that the treated cells could be labeled by Alexa Fluor 488 annexin V (SUPplementary Figure 3C) in a similar way to Figure 3B. With the laser power further reduced, the cell death efficiency decreased to less than 50\% (Figure 3D). MTT assay was also performed to quantify the cell death and the result is shown in Figure 3E. After laser irradiation at $2.18 \mathrm{~W} / \mathrm{cm}^{2}$, the O.D. at $570 \mathrm{~nm}$ was reduced by $54.8 \%$ for macrophages with nanorods, but only by $9.4 \%$ for the control group without nanorods. These results together confirmed that nanorod-mediated optical hyperthermia could effectively eradicate activated macrophages in the apoptosis or necrosis pathway by controlling laser irradiation condition, and we used $2.18 \mathrm{~W} /$ $\mathrm{cm}^{2}(0.43 \mathrm{~W})$ in our later study as the optimized condition to induce apoptosis.

We further investigated the mechanisms underlying the observed necrosis and apoptosis. In previous photothermal therapy studies, damage of the plasma membrane by irradiation of nanoparticles $[10,12]$ and gold nanorods [26] was reported. Therefore, we first examined the plasma membrane integrity and $\mathrm{Ca}^{2+}$ influx into cells under the necrosis and apoptosis conditions. We incubated the macrophages with Oregon Green BAPTA 2, a common $\mathrm{Ca}^{2+}$ indicator, and washed the cells before laser irradiation. The control experiment was performed with macrophages without nanorods to estimate the percentage of photobleaching. After laser irradiation at $4.36 \mathrm{~W} / \mathrm{cm}^{2}$ for necrosis or $2.18 \mathrm{~W} / \mathrm{cm}^{2}$ for apoptosis, the intracellular intensity of Oregon Green BAPTA 2 was compared. As shown in Figure 4, the intensity level of Oregon Green was similar in the control and the apoptotic condition, while demonstrating an obvious increase during necrotic conditions. These results indicate that loss of plasma membrane integrity and $\mathrm{Ca}^{2+}$ influx occurred in necrosis but not in apoptosis during the nanorod-mediated photothermolysis.

We have studied the role of intracellular injury in the induction of apoptosis. At a molecular level there are two principal mechanisms for apoptotic cell death, extrinsic (mitochondria-independent apoptosis) and intrinsic (mitochondria-dependent apoptosis) [50]. Therefore, we examined whether mitochondria were damaged by nanorod-mediated optical hyperthermia. After laser irradiation of nanorod-internalized macrophages at $2.18 \mathrm{~W} / \mathrm{cm}^{2}$ for 15 min, Mito Tracker Red CMXRos and Alexa Fluor 488 annexin $V$ were used to detect mitochondrial membrane potential change [51] and indicate apoptosis, respectively. In comparison with nonradiated macrophages, the fluorescent intensity of Mito Tracker Red decreased by 70\% in apoptotic macrophages, but by only $16.8 \%$ in the control group without nanorod labeling (Figure 5A-C). Morphological change of mitochondria was also detected at the same condition with labeling of Mito Tracker green FM. As shown in Figure 5D, both mitochondrial fission and swelling were observed. Similar results were also found in the TEM images shown in Figure 5E \& $\mathrm{F}$. In addition, we also detected an overproduction of reactive oxygen species in the apoptotic macrophages after laser irradiation (FIgure 6), a sign 
of oxidative stress. These observations provide further evidence of mitochondrial damage [52]. Together, the results suggested a mitochondriadependent apoptosis pathway for nanorod-mediated photothermolysis of macrophages.

The detailed pathway of mitochondrial damage is beyond the scope of the current paper. We anticipate that irradiation of gold nanorods and local energy deposition could generate heat and cavitation, in other words, bubble formation and collapse $[12,53]$. The localized heat and cavitation might cause cellular stress and disruption of some intracellular compartments such as the endoplasmic reticulum. These intracellular perturbations and signals released could lead to the formation of mitochondrial permeability transition pores [54], causing membrane potential decrease and mitochondrial swelling. Upon the permeability transition, apoptogenic factors (e.g., cytochrome c) could leak into cytoplasm and trigger a cascade of apoptotic cell death.

\section{Conclusion}

The current work demonstrated that gold nanorods conjugated with arginine-rich peptides could be selectively internalized by activated macrophages in vitro and in vivo without the assistance of antibody- or ligand-mediated targeting. Utilizing gold nanorods as efficient optothermal energy converters, apoptosis of macrophages was induced in a mitochondria-dependent pathway during nanorod-mediated optical hyperthermia under the optimized laser power density of $2.18 \mathrm{~W} / \mathrm{cm}^{2}$. As activated macrophages are becoming a valid pharmaceutical target of the autoimmune and inflammatory diseases, optical hyperthermia using gold nanorods provides a promising alternative to theragnose the activated macrophages and to treat the related diseases. The controlled cell death and better understanding of the mechanism facilitate the translation of the laboratory innovation to the clinic.

\section{Future perspective}

With the development of catheter-based optical fiber, lasers have been used in clinical therapy to ablate atherosclerotic lesions. Taking the advantages of selective uptake and localized hyperthermia of macrophages mediated by gold nanorods, the efficiency and accuracy of laser surgery could be greatly improved. As demonstrated in our work, cell death could be induced in an apoptosis pathway to reduce the inflammatory effect caused by necrosis.

Several issues have to be considered when pushing the application to the in vivo level. First, the surface coating needs to be designed to ensure elongated circulation of gold nanorods in blood and sufficient accumulation in the lesion area. As shown in Figure 1, octaarginine peptides could increase the efficiency of internalization of gold nanorods in macrophages, while coating with polyethylene glycol (PEG) could reduce uptake by macrophages. Therefore, gold nanorods with both PEG protection and specific ligands to trigger efficient uptake by macrophages at the site of injury could be applicable. Second, although colloid gold has been used in vivo since the 1950s [55], it remains unknown whether gold nanorods cause organ toxicity. The viability of cultured cells after long-term incubation of gold nanorods might not represent what will occur in the in vivo environment. These issues are being addressed by investigating the pharmacokinetic behavior and toxicity of functionalized gold nanorods in animal models in our laboratory.

\section{Acknowledgments}

The authors thank WXia for help in preparation of macrophages, and A Wei for critical reading of the manuscript.

Financial \& competing interests disclosure

This work was supported by NSF grant CBET 0828832 to J-X Cheng and American Heart Association Greater Midwest Predoctoral fellowship to L Tong. The authors

\section{Executive summary}

\section{Efficient uptake of cysteine-octaarginine-conjugated gold nanorods by activated macropahges}

- Conjugated with arginine-rich peptides, gold nanorods could be efficiently internalized by primary activated macrophages in vitro and in live animals.

- Cellular uptake of gold nanorods is monitored by two-photon luminescence.

\section{Apoptosis \& necrosis induced by nanorod-mediated photothermolysis}

- Apoptosis or necrosis to nanorod-internalized macrophages could be induced by controlling the laser irradiation condition.

- The current study demonstrates a promising method to treat inflammatory diseases via photothermal elimination of activated macrophages.

\section{Mechanisms of photothermolysis}

- Apoptosis was induced by intracellular mitochondrial damage. Reactive oxygen species release, mitochondrial membrane potential drop, and mitochondrial swelling were observed.

Necrosis was induced by disruption of plasma membrane integrity and subsequent $\mathrm{Ca}^{2+}$ influx. 
have no other relevant affiliations or financial involvement with any organization or entity with a financial interest in or financial conflict with the subject matter or materials discussed in the manuscript apart from those disclosed.

No writing assistance was utilized in the production of this manuscript.

\section{Ethical conduct of research}

The authors state that they have obtained appropriate institutional review board approval or have followed the principles outlined in the Declaration of Helsinki for all human or animal experimental investigations. In addition, for investigations involving human subjects, informed consent has been obtained from the participants involved.

\section{Bibliography}

1 Libby P: Inflammation in atherosclerosis. Nature 420, 868-874 (2002).

2 Kinne RW, Brauer R, Stuhlmuller B, Palombo-Kinne E, Burmester GR: Macrophages in rheumatoid arthritis. Arthritis Res. 2, 189-202 (2000).

3 Zissel G, Prasse A, Muller-Quernheim J: Sarcoidosis-immunopathogenetic concepts. Semin. Respir. Crit. Care Med. 28, 3-14 (2007).

4 Gordon S: Alternative activation of macrophages. Nat. Rev. Immunol. 3, 23-35 (2003).

5 Li AC, Glass CK: The macrophage foam cell as a target for therapeutic intervention. Nat. Med. 8, 1235-1242 (2002).

6 Moghimi SM, Hunter AC, Murray JC: Nanomedicine: current status and future prospects. FASEB J. 19, 311-330 (2005).

7 Moghimi SM, Bonnemain B: Subcutaneous and intravenous delivery of diagnostic agents to the lymphatic system: applications in lymphoscintigraphy and indirect lymphography. Adv. Drug Deliv. Rev. 37, 295-312 (1999).

8 Agrawal AK, Gupta CM: Tuftsin-bearing liposomes in treatment of macrophage-based infections. Adv. Drug Deliv. Rev. 41, 135-146 (2000).

9 Gabizon AA, Shmeeda H, Zalipsky S: Pros and cons of the liposome platform in cancer drug targeting. J. Liposome Res. 16, 175-183 (2006).

10 Pitsillides CM, Joe EK, Wei X, Anderson RR, Lin CP: Selective cell targeting with light-absorbing microparticles and nanoparticles. Biophys. J. 84, 4023-4032 (2003).

11 O'Neal DP, Hirsch LR, Halas NJ, Payne JD, West JL: Photo-thermal tumor ablation in mice using near infrared-absorbing nanoparticles. Cancer Lett. 209, 171-176 (2004).

12 Zharov VP, Letfullin RR, Galitovskaya EN: Microbubbles-overlapping mode for laser killing of cancer cells with absorbing nanoparticle clusters. J. Phys. D 38, 2571-2581 (2005).
13 El-Sayed IH, Huang X, El-Sayed MA: Selective laser photo-thermal therapy of epithelial carcinoma using anti-EGFR antibody conjugated gold nanorparticles. Cancer Lett. 239, 125-139 (2005).

14 Hirsch LR, Stafford RJ, Bankson JA et al.: Nanoshell-mediated near-infrared thermal therapy of tumors under magnetic resonance guidance. Proc. Natl Acad. Sci. USA 100, 13549-13554 (2003).

15 Loo C, Lowery A, West J, Halas N, Drezek R: Immunotargeted nanoshells for integrated cancer imaging and therapy. Nano Lett. 5 , 709-711 (2005).

16 Au L, Zheng D, Zhou F, Li Z-Y, Li X, Xia Y: A quantitative study on the photothermal effect of immuno gold nanocages targeted to breast cancer cells. ACS Nano. 2, 1645-1652 (2008).

17 Chen J, Wang D, Xi J et al.: Immuno gold nanocages with tailored optical properties for targeted photothermal destruction of cancer cells. Nano Lett. 7, 1318-1322 (2007).

18 Nikoobakht B, El-Sayed MA: Preparation and growth mechanism of gold nanorods (NRs) using seed-mediated growth method. Chem. Mater. 15, 1957-1962 (2003).

19 Sau TK, Murphy CJ: Seeded high yield synthesis of short Au nanorods in aqueous solution Langmuir 20, 6414-6420 (2004).

20 Liao H, Hafner JH: Gold nanorod bioconjugation. Chem. Mater. 17, 436-4641 (2005).

21 Yu C, Irudayaraj J: Multiplex biosensor using gold nanorods. Anal. Chem. 79, 572-579 (2007).

22 Huang X, El-Sayed IH, Qian W, El-Sayed MA: Cancer cells assemble and align gold nanorods conjugated to antibodies to produce highly enhanced, sharp, and polarized surface Raman spectra: a potential cancer diagnostic marker. Nano Lett. 7 , 1591-1597 (2007).

23 Durr NJ, Larson T, Smith DK, Korgel BA, Sokolov K, Ben-yakar A: Two-photon luminescence imaging of cancer cells using molecularly targeted gold nanorods. Nano Lett. 7, 941-945 (2007).
24 Huff TB, Hansen MN, Zhao Y, Cheng J-X, Wei A: Controlling the cellular uptake of gold nanorods. Langmuir 23, 1596-1599 (2007).

25 Huff TB, Tong L, Zhao Y, Hansen MN, Cheng J-X, Wei A: Hyperthermic effects of gold nanorods on tumor cells. Nanomed. 2, 125-132 (2007).

26 Tong L, Zhao Y, Huff TB, Hansen MN, Wei A, Cheng J-X: Gold nanorods medicate tumor cell death by compromising membrane integrity. Adv. Mater. 19, 3136-3141 (2007).

27 Takahashi H, Niidome Y, Niidome T, Kaneko K, Kawasaki H, Yamada S: Modification of gold nanorods using phosphatidylcholine to reduce cytotoxicity. Langmuir 22, 2-5 (2006).

28 Chen C-C, Lin Y-P, Wang C-W et al. DNA-gold nanorods conjugates for remote control of localized gene expression by near infrared irradiation. J. Am. Chem. Soc. 128, 3709-3715 (2006).

29 Oyelere AK, Chen PC, Huang X, El-Sayed IH, El-Sayed MA: Peptideconjugated gold nanorods for nuclear targeting. Bioconjug. Chem. 18, 1490-1497 (2007).

30 Niidome T, Yamagata M, Okamoto Y et al. PEG-modified gold nanorods with a stealth character for in vivo applications. J. Control. Release 114, 343-347 (2006).

31 Hotchkiss JW, Lowe AB, Boyes SG: Surface modification of gold nanorods with polymers synthesized by reversible additionfragmentation chain transfer polymerization. Chem. Mater. 19, 6-13 (2007).

32 Hauck TS, Ghazani AA, Chan WCW: Assessing the effect of surface chemistry on gold nanorod uptake, toxicity, and gene expression in mammalian cells. Small 4, 153-159 (2008).

33 Sonnichsen C, Alivisatos AP: Gold nanorods as novel nonbleaching plasmon-based orientation sensors for polarized singleparticle microscopy. Nano Lett. 5, 301-304 (2005).

34 Murphy CJ, Gole AM, Hunyadi SE et al.: Chemical sensing and imaging with metallic nanorods. Chem. Commun. 5, 544-557 (2008). 
35 Wang $\mathrm{H}$, Huff $\mathrm{TB}$, Zweifel DA et al.: In vitro and in vivo two-photon luminescence imaging of single gold nanorods. Proc. Natl Acad. Sci. USA 102, 15752-15756 (2005)

36 Imura K, Nagahara T, Okamoto $\mathrm{H}$ : Near-field two-photon-induced photoluminescence from single gold nanorods and imaging of plasmon modes. J. Phys. Chem. B 109, 13214-13220 (2005).

37 Link S, El-Sayed MA: Spectral properties and relaxation dynamics of surface plasmon electronic oscillations in gold and silver nanodots and nanorods. J. Phys. Chem. B 103, 8410-8426 (1999).

38 Chou C-H, Chen C-D, Wang CRC: Highly efficient, wavelength-tunable, gold nanoparticle based optothermal nanoconvertors. J. Phys. Chem. B 109, 11135-11138 (2005).

39 Huang X, El-Sayed IH, Qian W, El-Sayed MA: Cancer cell imaging and photothermal therapy in the near-infrared region by using gold nanorods. J. Am. Chem. Soc. 128, 2115-2120 (2006).

40 Jain PK, Lee KS, El-Sayed IH, El-Sayed MA: Calculated absorption and scattering properties of gold nanoparticles of different size, shape, and composition: applications in biological imaging and biomedicine. J. Phys. Chem. B 110, 7238-7248 (2006).
41 Takahashi H, Niidome Y, Yamada S: Controlled release of plasmid DNA from gold nanorods induced by pulsed near-infrared light. Chem. Commun. 17, 2247-2249 (2005).

42 Murphy CJ, Gole AM, Stone JW et al.: Gold nanoparticles in biology: beyond toxicity to cellular imaging. Acc. Chem. Res. 41(12), 1721-1730 (2008).

43 Takahashi H, Niidome T, Nariai A, Niidome Y, Yamada S: Gold nanorodsensitized cell death: Microscopic observation of single living cells irradiated by pulsed near-infrared laser light in the presence of gold nanorods. Chem. Lett. 35, 500-501 (2006).

44 Pissuwan D, Valenzuela SM, Information C et al:: Targeted destruction of murine macrophage cells with bioconjugated gold nanorods. J. Nanoparticle Res. 9, 1109-1124 (2007).

45 Norman RS, Stone JW, Gole A, Murphy CJ, Sabo-Attwood TL: Targeted photothermal lysis of the pathogenic bacteria, pseudomonas aeruginosa, with gold nanorods. Nano Lett. 8 , 302-306 (2008).

46 He W, Henne WA, Wei Q et al:: Two-photon luminescence imaging of bacillus spores using peptide-functionalized gold nanorods. Nano Res. 1, 450-456 (2008).

47 Zweifel DA, Wei A: Sulfide-arrested growth of gold nanorods. Chem. Mater. 17, 4256-4261 (2005).
48 Orendorff CJ, Murphy CJ: Quantitation of metal content in the silver-assisted growth of gold nanorods. J. Phys. Chem. B 110, 3990-3994 (2006).

49 Li T, Hu J, Thomas JA, Li L: Differential induction of apoptosis by LPS and taxol in monocytic cells. Mol. Immunol. 42, 1049-1055 (2005).

50 Fulda S, Debatin KM: Extrinsic versus intrinsic apoptosis pathways in anticancer chemotherapy. Oncogene 25, 4798-4811 (2006).

51 Poot M, Pierce RH: Detection of changes in mitochondrial function during apoptosis by simultaneous staining with multiple fluorescent dyes and correlated multiparameter flow cytometry. Cytometry 35, 311-317 (1999).

52 Mignotte B, Vayssiere JL: Mitochondria and apoptosis. Eur. J. Biochem. 252, 1-15 (1998).

53 Lin CP, Kelly MW: Cavitation and acoustic emission around laser-heated microparticles. Appl. Phys. Lett. 72, 2800-2802 (1998).

54 Crompton M: The mitochondrial permeability transition pore and its role in cell death. Biochem. J. 341, 233-249 (1999).

55 Sherman AI, Ter-Pogossian M: Lymph-node concentration of radioactive colloidal gold following interstitial injection. Cancer 6 , 1238-1240 (1953). 